\title{
Understanding Room Temperature Deformation of High-Strength Titanium Alloys
}

John Foltz ${ }^{1}$, Shaolou $\mathrm{Wei}^{2}$, Luis Ruiz-Aparicio ${ }^{3}$, Andy Martinez ${ }^{3}$ and C. Cem Tasan ${ }^{2}$

${ }^{1}$ ATI Specialty Materials, Monroe, North Carolina, United States, ${ }^{2}$ Massachusetts Institute of Technology, Cambridge, Massachusetts, United States, ${ }^{3}$ ATI Specialty Rolled Products, Natrona Heights, Pennsylvania, United States

Metallic sheets are used in a variety of consumer and commercial applications due to their lower cost and wide applicability for manufacturing. Titanium alloys are often limited in these application due to difficulty processing large ingots into sheet form, which stems from limited ductility near room temperature to support large strains encountered by cold rolling. Exceptions to this do exist, however, and alloys like ATI $425^{\circledR}$ titanium alloy have both high strength and cold ductility. The underlying reason for this desired property combination has not been fully understood given its beta fraction $(\sim 5 \%)$ and higher oxygen content (e.g. than that of Ti-6Al-4V) which in unalloyed titanium is known to reduce cold ductility [1].

To discover the metallurgical reasons for the surprising cold ductility of ATI $425^{\circledR}$ titanium alloy, ATI examined deformation characteristics of three titanium alloys in multiple microstructural conditions using EBSD slip-trace analysis. This was done by first polishing bulk specimens taken from rolled or drawn bar, performing bulk compression tests, and then analyzing the pre-polished surfaces with EBSD while simultaneously imaging with secondary electrons. Surface reliefs on the polished surface correlated with dislocation shear bands, and EBSD uncovered the crystallographic nature of the shear plane it fell on as well as identifying the presence of twining. By comparing the different alloys in two microstructural conditions, it was observed that bimodal microstructures resulted in more intense strain localization, and was consistent with existing literature that showed highly alloyed samples demonstrate reduced twinning [2].

Further characterization work was done at MIT using in-situ tensile testing on sheet samples. Testing these samples in different loading conditions led to more insights regarding the deformation mechanisms in ATI $425^{\circledR}$ titanium alloy. Specifically, in situ observations show that unfavorably aligned grains with low Schmid factor in ATI $425^{\circledR}$ titanium alloy sheet routinely exhibit a twinning response that rotates the twinned region to a higher Schmid factor. This results in a higher critically resolved shear stress on the slip systems that can be easily activated at room temperature, such as A-type basal, prism, and pyramidal systems, and $\mathrm{C}+\mathrm{A}$ type pyramidal systems. The twinned region is then capable of additional plastic deformation. This is a surprising discovery, as higher oxygen content in titanium alloys is known to suppress twinning activation. Aluminum is also known to reduce twinning response, and compared with other high-strength titanium alloys, ATI $425^{\circledR}$ titanium alloy has lower aluminum. Differences in the deformation response, along with the microstructural differences between ATI 425® Alloy and Ti-6Al$4 \mathrm{~V}$ explain the majority of the observed differences in cold ductility. Future work will be needed to understand the limits of twinning activation in the alloy compare with other high-strength alpha-beta titanium alloys. 


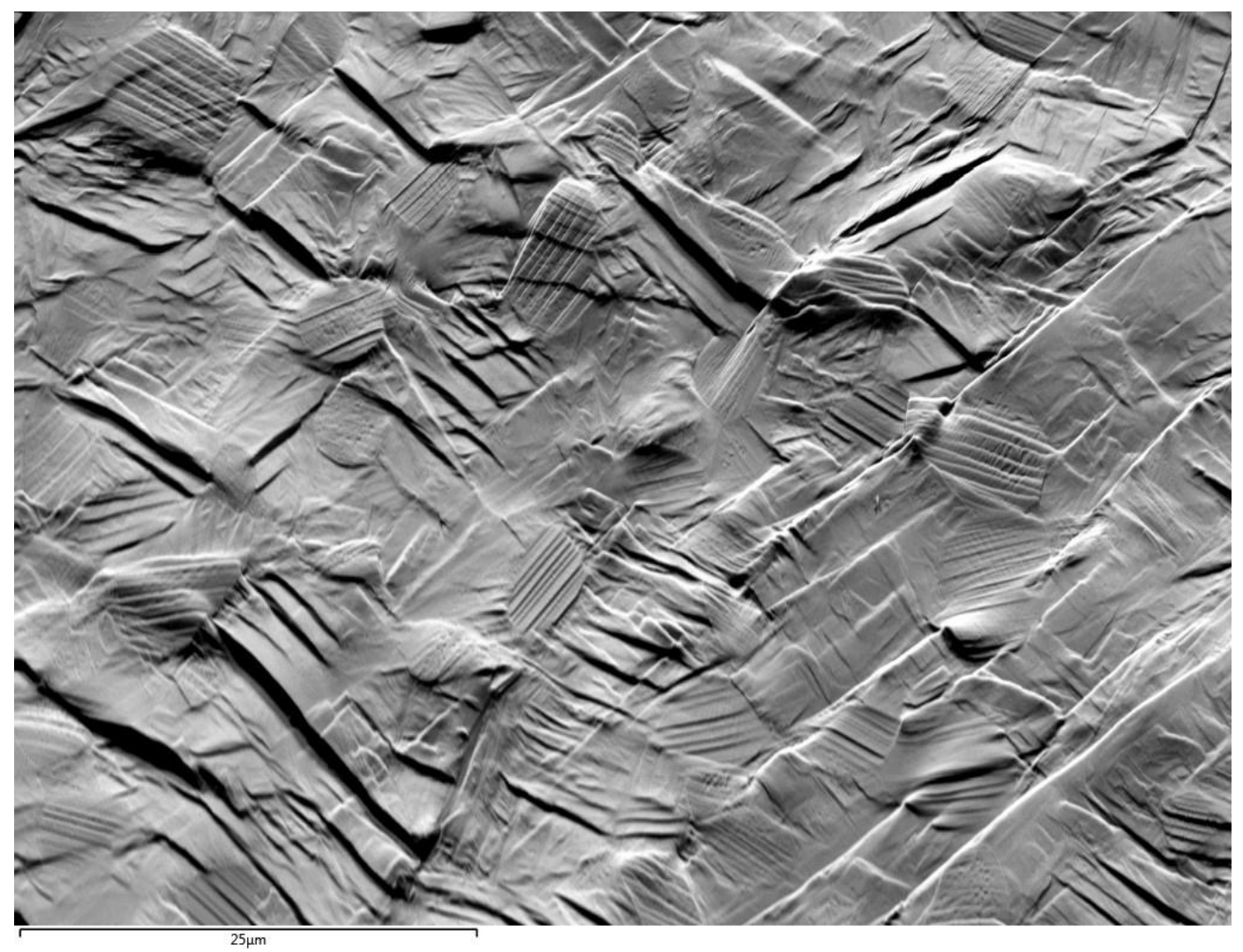

Figure 1. Secondary electron image of ATI 6-4TM Alloy wire compressed along axial direction after being pre-polished for EBSD analysis, which combined with imaging can be linked to dislocation slip systems.


Figure 2. Tensile twin observed in ATI $425 ®$ Alloy sheet that rotates the grains to a higher critically resolved shear stress, which activates additional dislocation slip systems and results in improved uniform elongation.

References

[1] G. Lütjering and J.C. Williams. Titanium. Springer. $2^{\text {nd }}$ Ed. 2007, p. 188.

[2] G. Lütjering and J.C. Williams. Titanium. Springer. $2^{\text {nd }}$ Ed. 2007, p. 19. 